\title{
RNA Interference Mediated Silencing of Tissue Factor in Human Umbilical Vein Endothelial Cells
}

\author{
Xiaojuan Yin*, Zhu Chen*, Weiqin Zhou, Wen Tang, Zhichun Feng\# \\ Bayi Children's Hospital Affiliated to Army General Hospital of People's Liberation Army, Beijing, China \\ Email: "drfengzc@fimmu.edu.cn
}

How to cite this paper: Yin, X.J., Chen, Z., Zhou, W.Q., Tang, W. and Feng, Z.C. (2018) RNA Interference Mediated Silencing of Tissue Factor in Human Umbilical Vein Endothelial Cells. Open Journal of Obstetrics and Gynecology, 8, 477-484. https://doi.org/10.4236/ojog.2018.85054

Received: December 13, 2017

Accepted: May 7, 2018

Published: May 10, 2018

Copyright ( 92018 by authors and Scientific Research Publishing Inc. This work is licensed under the Creative Commons Attribution International License (CC BY 4.0).

http://creativecommons.org/licenses/by/4.0/

\begin{abstract}
Objective: To construct to silence tissue factor (TF) expression in Human Umbilical Vein Endothelial Cells (HUVECs) for providing evidence of the gene therapy and prevention of coagulation dysfunction in placental abruption (PA) neonates. Methods: Cultured HUVECs in vitro were divided into control group and PA group, each including non-prevention, scramble siRNA and TF-siRNA subgroups. pENTRTM/U6-shRNA/TF vector expressing TF were constructed and transfected into HUVECs. The mRNA expression of TF was tested with RT-PCR, and TF protein expression was detected with immunofluorescence staining. Results: Monolayer HUVECs with short-rod and short spindle shaped were adherent to the bottom, forming paving stone arrangement. The TF mRNA expression was significantly different between control group and PA group $(P<0.01)$ and among different subgroups $(P<$ $0.01)$. In non-prevention subgroups, significant difference was observed in TF mRNA expression between control group and PA group $(P<0.05)$. Significant difference in TF mRNA expression was found in false-prevention subgroups $(P<0.05)$. The TF mRNA expression was markedly different among different subgroups in control $(P<0.01)$, and the similar result among different PA subgroups $(P<0.01)$. In both control and PA groups, the TF mRNA expression was the lowest after TF silencing. Immunofluorescence staining showed high TF expression in HUVECs in false-prevention subgroups. In scramble siRNA group, the TF protein expression reduced as compared to non-prevention group and reduced dramatically after TF silencing as compared to control. Conclusion: $\mathrm{pENTR}^{\mathrm{TM}} / \mathrm{U} 6-T F-$ shRNA is able to significantly inhibit the TF mRNA and protein expression in HUVECs from healthy neonates and PA neonates.
\end{abstract}

${ }^{\star}$ Contributed equally. 


\section{Keywords}

Neonate, Tissue Factor, Placental Abruption

\section{Introduction}

Placental abruption (PA) refers to the complete or partial separation of a normal placenta from the wall of the uterus before delivery. PA is not only a major cause of bleeding but an important cause of coagulation dysfunction in neonates after birth in the Department of Obstetrics [1] [2]. PA may cause stillbirth, premature delivery, neonatal asphyxia, neonatal anemia and coagulation disorders. The PA related coagulation disorders are closely associated with some complications such as early DIC, scleroderma, intracranial hemorrhage and pulmonary hemorrhage. Previous studies reported that the incidence of PA is $0.49 \%-1.8 \%$ [3] [4]. In China, the incidence of PA is $0.46 \%-0.21 \%$ and PA accounts for $10 \%$ - $20 \%$ of deaths in perinatal period [5]. Studies have shown that a large amount of tissue factor (TF) is released from the placental villi and decidua in PA and forms TF-VII complex with fetal F-VII. The activated TF-VII complex may induce coagulation cascade, resulting in abnormal coagulation and finally disseminated intravascular coagulation (DIC) [6] [7] [8]. TF expression occurs at the initiation of coagulation, and inhibition of TF expression has the possibility to reduce the risk for coagulation dysfunction in PA neonates. In the present study, RNA interference (RNAi) [9] was employed to construct a vector expressing TF which was then transfected into human umbilical vein endothelial cells (HUVECs) to silence TF expression. Our findings may provide a new way for the clinical therapy and prevention of coagulation dysfunction in PA neonates.

\section{Materials and Methods}

\subsection{Main Materials}

RPMI 1640 medium, collagenase II, trypsin (Gibco), fetal bovine serum (Hyclone), epidermal growth factor (Peprotec), VIII detection kit (Antibody Diagnotica), anti-human TF monoclonal antibody (R \& D Systems, Inc), goat anti-rabbit immunofluorescene antibody (Zhongshan Golden Bridge Biotech Co., Ltd), BLOCK-iT ${ }^{\mathrm{TM}}$ U6 RNAi Entry Vector kit, Gene Juice Transfection Reagent Novagen (Invitrogen), sequences for TF silencing (Shanghai Sangong Biotech Co., Ltd.), plasmid extraction Midi Kit (Qiagen) and other domestic analytically pure reagents were used in this study.

\subsection{Methods}

\subsubsection{In Vitro Culture and Identification of HUVECs}

The umbilical cord (about $10 \mathrm{~cm}$ ) was collected in an aseptical condition during delivery, and the blood in the umbilical cord was removed by flushing the umbilical cord. Then, $0.1 \%$ collagenase II $(5 \mathrm{ml})$ was added, followed by digestion 
for $12 \mathrm{~min}$ at $37^{\circ} \mathrm{C}$. The solution was harvested after digestion, and the umbilical cord was flushed again with PBS. After addition of FBS, centrifugation was done, and the supernatant was removed. The cells were re-suspended in complete medium and then seeded into $25-\mathrm{cm}^{2}$ dish, followed by incubation at $37^{\circ} \mathrm{C}$ in an environment with $5 \% \mathrm{CO}_{2}$ for $24 \mathrm{~h}$. Subsequently, epidermal growth factor (EGF) was added, followed by incubation for $48-72 \mathrm{~h}$. Immunohistochemistry was done for the measurement of VIII protein expression. Cells of $2^{\text {nd }}$ and $3^{\text {rd }}$ generation were harvested for following experiments.

\subsubsection{Construction and Identification of pENTRTM/U6-shRNA/TF}

According to the interference sequences T12 and T6 of TF, shRNA sequence was designed as follow:

\section{CACCGTTCCTTCTGACTAAAGTCCGTCCGTCGAAACGGACTTTAGTCA}

GAAGGAA, and ds oligo were synthesized. After annealing, the ds oligo was connected to the sticky ends of $\mathrm{pENTR}^{\mathrm{TM}} / \mathrm{U} 6$. The resultant products were transformed into competent cells to construct pENTRTM/U6-shRNA/TF. After sequencing, the vectors were used in following experiments.

\subsubsection{Detection of TF mRNA and Protein Expression in HUVECs after Transfection}

HUVECs were divided into normal control group and PA group, both of which were further subdivided into 3 subgroups independently: 1) non-transfection group; 2) scramble shRNA transfection group; 3) TF shRNA transfection group ( $n=3$ per group). The mRNA and protein expression of TF were measured before and after transfection.

1) Transfection: HUVECs were seeded into 12 -well plates at a density of $5 \times$ $10 \% / \mathrm{ml}$. When the cell confluence reached $60 \%-80 \%, 0.5 \mu \mathrm{g}$ of plasmid DNA was added, and pENTRTM/U6-shRNA/TF was transfected into HUVECs after incubation at $37^{\circ} \mathrm{C}$ for $48 \mathrm{~h}$.

2) Detection of TF mRNA expression by RT-PCR: The supernatant in 12-well plates was removed and two step RT-PCR was performed to detect TF mRNA expression. The conditions for reverse transcriptions were as follows: $42^{\circ} \mathrm{C}$ for $20 \mathrm{~min}, 95^{\circ} \mathrm{C}$ for $5 \mathrm{~min}$ and $5^{\circ} \mathrm{C}$ for $5 \mathrm{~min}$. Primers were designed according to the human TF cDNA sequence (NM_001993):

5'-CCGGCACAGCTTTAACAACCT-3' (forward) and 5'-CGTTTGCTCTCGATTCCATGTG-3' (reverse). Human $\beta$-actin gene (CF62602) served as an internal reference and its primers were as follows: 5'-TGGCACCACACCTTCTACAATG-3' (forward) and 5'-CCGTGGTGGTGAAGCTGTAGC-3' (reverse). The conditions for PCR were as follows: $94^{\circ} \mathrm{C}$ for $5 \mathrm{~min}, 32 \mathrm{cycles}$ at $94^{\circ} \mathrm{C}$ for $30 \mathrm{~s}, 50^{\circ} \mathrm{C}$ for $30 \mathrm{~s}$ and $72^{\circ} \mathrm{C}$ for $30 \mathrm{~s}$ and a final extension at $72^{\circ} \mathrm{C}$ for $5 \mathrm{~min}$. The products were subjected to $1.5 \%$ agarose gel electrophoresis, and visualization was done with Bio-rad gel image system. Bands were analyzed with Gel-Pro Analyzer, and the optical density was determined as the mRNA expression. The mRNA expression of TF was normalized to that of $\beta$-actin. 
3) Detection of TF protein expression by immunofluorescence staining: Coverslips sized $1 \mathrm{~cm} \times 1 \mathrm{~cm}$ were placed into 12-well plates and then HUVECs were seeded into 12 -well plates at a density of $5 \times 10^{4} / \mathrm{ml}$. When the cell confluence reached $60 \%-80 \%$, the vectors were transfected into HUVECs. The supernatant was removed, and cells were washed with PBS. After air-drying, cells were fixed in paraformaldehyde at room temperature. Rabbit anti-human TF antibody (1:200) was added, followed by incubation in a humidified environment. Following washing in PBS thrice, FITC conjugated goat anti-rabbit secondary antibody (1:400) was added, followed by incubation for $2 \mathrm{~h}$. Cells were observed under a fluorescence microscope.

\subsubsection{Statistical Analysis}

Statistical analysis was performed with SPSS version 17.0. Quantitative data are expressed as mean \pm standard deviation $(X \pm S D)$, and categorical variables as percentages and tested with chi square test or Fisher's exact test if necessary. Quantitative data were compared with independent sample $t$ test between two groups and one way analysis of variance among groups. A value of $P<0.05$ was considered statistically significant.

\section{Results}

\subsection{In Vitro Culture and Identification of HUVECs}

Under an inverted microscope, monolayer HUVECs were adherent to the bottom, and short-rod like or short spindle shaped. The nucleus was oval, and nucleolus was occasionally observed. Cells formed paving stone arrangement. Immunohistochemistry for VIII showed cells had green fluorescence (Figure 1).

\subsection{TF mRNA Expression in HUVECs}

One way analysis of variance showed there was significant difference in the TF mRNA expression between control group and PA group $(F=23.436, P<0.01)$. Among different treatment groups, the TF mRNA expression was also significantly different $(F=33.630, P<0.01)$. In non-transfection subgroups, the TF mRNA expression was significantly different between control group and PA group ( $t=11.71, P<0.05$ ); in scramble shRNA transfection subgroups, the TF mRNA expression was significantly different between control group and PA group ( $t=21.112, P<0.05$ ); in TF shRNA transfection subgroups, the TF mRNA expression was comparable between control group and PA group $(\mathrm{t}=$ $0.023, P>0.05)$. In subgroups of control groups, the TF mRNA expression was also significant different $(F=27.657, P<0.01)$; in subgroups of PA groups, the TF mRNA expression was also significant different $(F=19.299, P<0.01)$; the TF mRNA expression was the lowest after TF silencing in both control groups and PA groups (Table 1).

\subsection{TF Protein Expression in HUVECs}

After immunofluorescence staining, HUVECs were found to have green 
Table 1. Relative TF mRNA expression in different groups $(\bar{x} \pm s)$.

\begin{tabular}{|c|c|c|c|c|c|c|}
\hline \multirow{2}{*}{ Group } & \multicolumn{3}{|c|}{ TF mRNA expression $\left(\begin{array}{ll}\bar{x} & \pm s\end{array}\right)$} & \multirow{2}{*}{ Total } & \multirow{2}{*}{$F$} & \multirow{2}{*}{$P$} \\
\hline & Non-transfection & Scramble shRNA & TF shRNA & & & \\
\hline $\mathrm{n}$ & 3 & 3 & 3 & & & \\
\hline Control & $0.657 \pm 0.097$ & $0.540 \pm 0.079$ & $0.220 \pm 0.030$ & $0.472 \pm 0.206$ & 27.657 & $<0.01$ \\
\hline PA & $1.323 \pm 0.323$ & $1.057 \pm 0.178$ & $0.207 \pm 0.150$ & $0.862 \pm 0.542$ & 19.299 & $<0.01$ \\
\hline Total & $0.990 \pm 0.4229$ & $0.798 \pm 0.309$ & $0.2133 \pm 0.097$ & $0.667 \pm 0.446$ & $33.630^{\star}$ & $<0.01^{\star}$ \\
\hline$t$ & 11.710 & 21.112 & 0.023 & $23.436^{*}$ & & \\
\hline$P$ & $<0.05$ & $<0.01$ & $>0.05$ & $<0.01^{*}$ & $6.555^{\#}$ & $0.012^{\#}$ \\
\hline
\end{tabular}

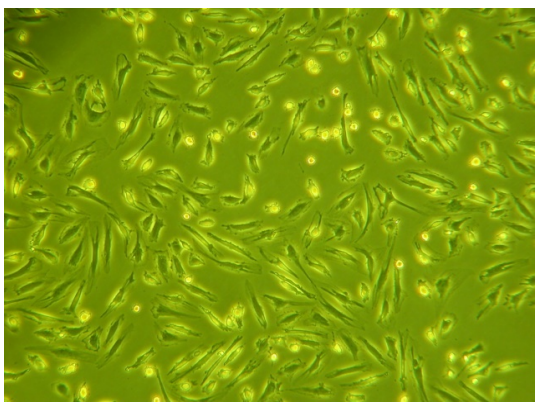

(a)

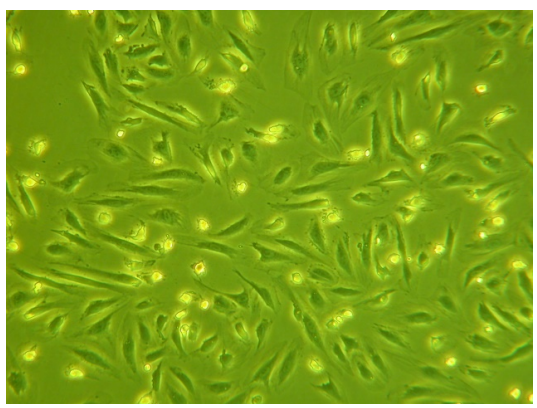

(c)

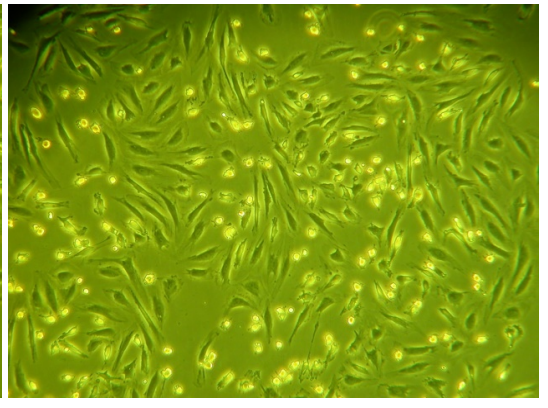

(b)

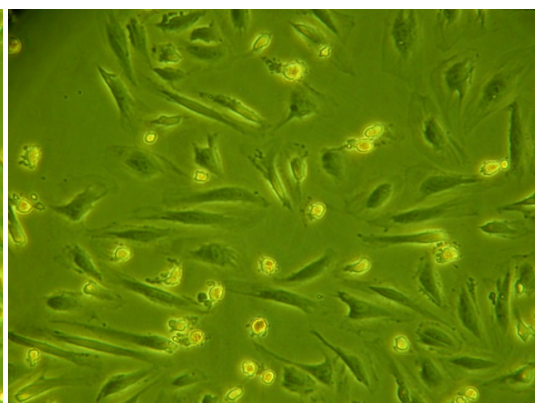

(d)

Figure 1. In vitro culture and identification of HUVECs. (a) Culture for $24 \mathrm{~h}(100 \times)$; (b) Culture for $48 \mathrm{~h}(100 \times)$; (c) Culture for $48 \mathrm{~h}(200 \times)$; (d) Culture for $48 \mathrm{~h}(200 \times)$.

fluorescence. In non-transfection group, strong fluorescence intensity of TF was found in HUVECs and the fluorescence was diffuse; in scramble shRNA transfection group, the TF protein expression reduced as compared to non-transfection group, and the fluorescence was sparse; in TF shRNA transfection group, the TF protein expression reduced dramatically as compared to control group, and the fluorescence was more sparse (Figure 2).

\section{Discussion}

PA may significantly increase the morbidity and mortality of neonates and thus has been paid increasing attention to [10] [11] [12]. In PA women, neonates may develop coagulation dysfunction [13], which significantly affects the coagulation and threaten the life. Thus, to find effective strategy to prevent and treat coagulation 


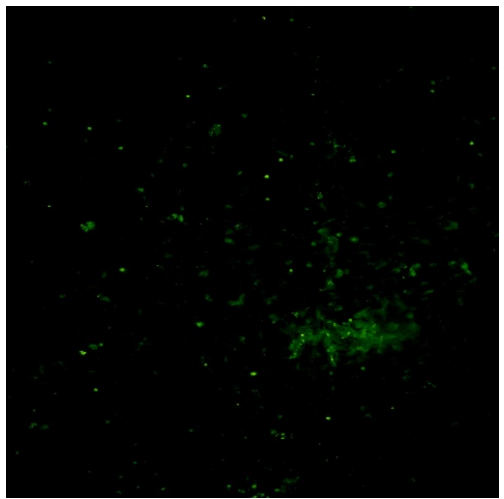

(a)

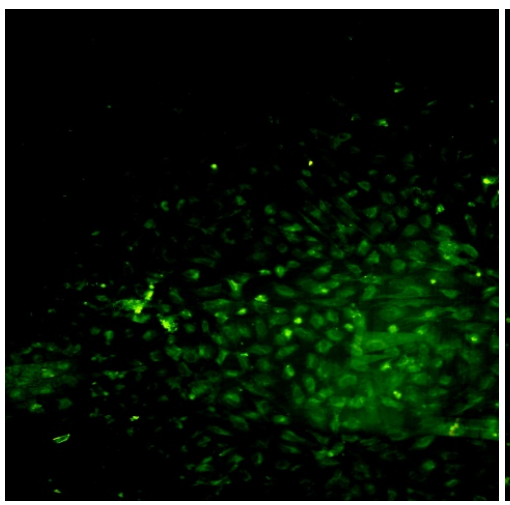

(b)

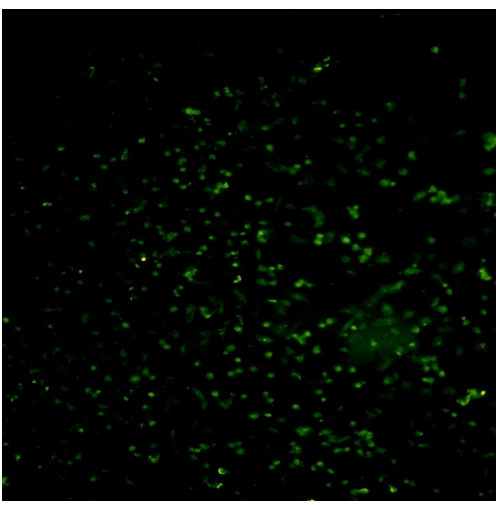

(c)

Figure 2. Immunofluorescence staining of TF protein in HUVECs TF. (a): TF shRNA transfection group (microscope: $100 \times)$; (b): scramble shRNA transfection group (200x); (c): non-transfection group (100X).

dysfunction related diseases in neonates is imperative. TF and tissue factor pathway inhibitor (TFPI) play crucial roles in the coagulation balance. In PA, the TF content of the placenta and myometrium is significantly higher than that in plasma, but the TFPI is a very low level [14] [15]. At the site of PA, placental villi and deciduas may release a large amount of TF which, on one hand, may enter the fetal circulation via the placenta, affecting the coagulation of fetus or neonates and, on the other hand, cause the coagulation activation in the fetus, leading to the coagulation disorders in the fetus or neonates. Under this condition, the thrombin increases dramatically and degrades fibrinogen into fibrin, causing DIC [16]. In the present study, results showed the mRNA and protein expression of TF in HUVECs from PA neonates increased significantly, suggesting that pathological PA may induce the up-regulation of TF expression. This indicates that the hypercoagulable state in PA women and their neonates is mainly ascribed to the entry of excess TF in the placenta and myometrium into the circulation, leading to the coagulation in neonates, which was consistent with previously reported [16].

RNAi technique is usually employed to efficiently and specifically down-regulate the expression of target gene and has been widely used in the studies on gene functions and gene therapy [9] [17]. In the present study, RNAi was used to silence TF expression in HUVECs and pENTR ${ }^{\mathrm{TM}} / \mathrm{U} 6-T F$-shRNA was successfully constructed because it could significantly inhibit the TF expression in HUVECs from PA neonates and healthy neonates, suggesting the silencing of TF expression. After transfection, the TF mRNA expression reduced dramatically in HUVECs from PA neonates and healthy neonates. The TF mRNA expression reduced by 3 folds in control group and 6 folds in PA group after transfection as compared to non-transfection group. This suggests TF-siRNA has more potent ability to inhibit TF expression in HUVECs from PA neonates. Immunofluorescence staining showed both protein and mRNA expression of TF reduced dramatically after TF-shRNA transfection. In addition, $\mathrm{pENTR}^{\mathrm{TM}} / \mathrm{U} 6-\mathrm{X}$-shRNA was able to inhibit the TF expression in HUVECs from both PA neonates and PA 
neonates. This suggests that $\mathrm{pENTR}^{\mathrm{TM}} / \mathrm{U} 6-\mathrm{X}$-shRNA may bind to the target site in a sequence independent manner, partially affecting TF mRNA expression, which was consistent with previously reported [18]. Torgeir found the transfection efficiency of siRNA was highly dependent on the target site of target gene. Thus, RNAi has limitations to different extents in studies on gene functions and drug development.

In the present study, $\mathrm{pENTR}^{\mathrm{TM}} / \mathrm{U} 6$ was used to successfully construct pENTRTM/U6-TF-shRNA which is then transfected into HUVECs from healthy neonates and PA neonates to inhibit the protein and mRNA expression of TF. Our findings support that silencing of TF expression is promising to block or attenuate the coagulation dysfunction in PA neonates and then reduce to the incidence of coagulation disorders in these neonates. In future studies, it is necessary to test the effects of TF expression silencing in vivo. In addition, RNAi has limitations in studies on gene function and drug development, and thus it is necessary to optimize this technique.

\section{References}

[1] Sakornbut, E., Leeman, L. and Fontaine, P. (2007) Late Pregnancy Bleeding. American Family Physician Journal, 75, 1199-1206.

[2] Wang, M. and Fontaine, P. (2014) Common Questions about Late-Term and Postterm Pregnancy. American Family Physician Journal, 90, 160-165.

[3] Roberts, J.M. (2014) Pathophysiology of Ischemic Placental Disease. Seminars in Perinatology, 38, 139-145. https://doi.org/10.1053/j.semperi.2014.03.005

[4] Tikkanen, M. (2011) Placental Abruption: Epidemiology, Risk Factors and Consequences. Acta Obstetricia et Gynecologica Scandinavica, 90, 140-149. https://doi.org/10.1111/j.1600-0412.2010.01030.x

[5] Le, J. (2004) Obstetrics and Gynecology. People's Health Publishing House, Beijing, 113-116.

[6] Hao, F., Wu, D.D., Xu, X. and Cui, M.Z. (2012) Histamine Induces Activation of Protein Kinase D That Mediates Tissue Factor Expression Abd Activity in Human Aortic Smooth Muscle Cells. American Journal of Physiology-Heart and Circulatory Physiology, 303, H1344-H1352. https://doi.org/10.1152/ajpheart.00500.2011

[7] Mackman, N. (2004) Role of Tissue Factor in Hemostasis, Thrombosis, and Vascular Development. Arteriosclerosis, Thrombosis, and Vascular Biology, 24, 1015-1022. https://doi.org/10.1161/01.ATV.0000130465.23430.74

[8] Laterre, P.F., Wittebole, X. and Collienne, C. (2006) Pharmacological Inhibition of Tissue Factor. Seminars in Thrombosis and Hemostasis, 32, 71-76. https://doi.org/10.1055/s-2006-933342

[9] Zhang, Q., Shu, F.L., Jiang, Y.F. and Huang, X.E. (2015) Influence of Expression Plasmid of Connective Tissue Growth Factor and Tissue Inhibitor of Metalloproteinase-1 shRNA on Hepatic Precancerous Fibrosis in Rats. Asian Pacific Journal of Cancer Prevention, 16, 7205-7210. https://doi.org/10.7314/APJCP.2015.16.16.7205

[10] Zhou, B.Y., Wu, X.L., Han, Y.K., et al. (2006) Characteristics of Neonates in Women with High Risk for Placental Abruption and Placenta Previa. Chinese Journal of Perinatal Medicine, 9, 48-50.

[11] Kupferminc, M.J., Rimon, E., Ascher-Landsberg, J., Lessing, J.B. and Many, A. 
(2004) Perinatal Outcome in Women with Severe Pregnancy Complications and Multiple Thrombophilias. Journal of Perinatal Medicine, 32, 225-227. https://doi.org/10.1515/JPM.2004.042

[12] Dafallah, S.E. and Babikir, H.E. (2004) Risk Factors Predisposing to Abruptio Placentae. Maternal and Fetal Outcome. Saudi Medical Journal, 25, 1237-1240.

[13] Sarwar, I., Abbasi, A.N. and Islam, A. (2006) Abruption Placentae and Its Complications at Ayub Teaching Hospital Abbottabad. Journal of Ayub Medical College Abbottabad, 18, 27-31.

[14] Kuczynski, J., Uszynski, W., Zekanowska, E., Soszka, T. and Uszyński, M. (2002) Tissue Factor (TF) and Tissue Factor Pathway Inhibitor (TFPI) in the Placenta and Myometrium. European Journal of Obstetrics \& Gynecology and Reproductive Biology, 105, 15-19. https://doi.org/10.1016/S0301-2115(02)00113-6

[15] Biguzzi, E., Franchi, F., Acaia, B., Ossola, W., Nava, U., Paraboschi, E.M., Asselta, R. and Peyvandi, F. (2014) Genetic Background and Risk of Postpartum Haemorrhage: Results from an Italian Cohort of 3219 Women. Haemophilia, 20, e377-e383.

[16] Wang, J.L., Xu, T.L. and Zhang, X.Y. (2004) Diagnosis and Treatment of Neonatal Disseminated Intravascular Coagulation Caused by Placental Abruption and Analysis of Relevant Factors. Chinese Journal of Pediatrics, 42, 391-392.

[17] Couzin, J. (2002) Breakthrongh of the Year. Small RNAs Make Big Splash. Science, 298, 2296-2301. https://doi.org/10.1126/science.298.5602.2296

[18] Holen, T., Amarzguioui, M., Wiiger, M.T., Babaie, E. and Prydz, H. (2002) Positional Effects of Short Interfering RNAs Targeting the Human Coagulation Trigger Tissue Factor. Nucleic Acids Research, 30, 1757-1766.

https://doi.org/10.1093/nar/30.8.1757 